\title{
Controlling granular segregation using modulated flow
}

\author{
Hongyi Xiao ${ }^{\mathrm{a}}$, David McDonald ${ }^{\mathrm{a}, \mathrm{b}}$, Yi Fan ${ }^{\mathrm{c}}$, Paul B. Umbanhowar ${ }^{\mathrm{a}}$, Julio M. Ottino ${ }^{\mathrm{a}, \mathrm{d}, \mathrm{e}}$, Richard M. Lueptow ${ }^{\mathrm{a}, \mathrm{d}, \mathrm{e}}$ \\ ${ }^{a}$ Department of Mechanical Engineering, Northwestern University, Evanston, Illinois 60208, USA \\ ${ }^{b}$ Department of Electrical and Computer Engineering (currently), Carnegie Mellon University, Pittsburgh, Pennsylvania 15213, USA \\ ${ }^{c}$ The Dow Chemical Company, Midland, Michigan 48667, USA \\ ${ }^{d}$ Department of Chemical and Biological Engineering, Northwestern University, Evanston, Illinois 60208, USA \\ ${ }^{e}$ The Northwestern Institute on Complex Systems (NICO), Northwestern University, Evanston, Illinois 60208, USA
}

\begin{abstract}
Unsteady flows of granular media are ubiquitous yet remain largely unexplored. In this research, we apply unsteady flows to strongly segregating granular materials to control the segregation pattern and enhance overall mixing. Sizebidisperse granular mixtures with large size ratios flowing onto a quasi-2D bounded heap form stratified layers of large and small particles when the flow rate is modulated. These layers exhibit better average mixing than the segregated patterns generated by steady feed rates. The mechanisms of layer formation under modulated flow differ from those for spontaneous stratification and are related to changes in the composition of the flowing layer at different stages in each feed cycle. The thickness and length of the stratified layers can be controlled by changing the feed rates and feed cycle durations, which is potentially useful for reducing segregation in industrial processes.
\end{abstract}

Keywords: Mixing and segregation, unsteady flows, stratification, flow modulation

\section{Introduction}

Segregation and mixing of disperse granular materials (differing in size, density, etc.) have important implications in situations ranging from material handling in industry to natural phenomena $[1,2]$. For example, in industry, segregation is frequently encountered in processes involving handling bulk solids such as ores, polymers, food, and pharmaceutical materials [3, 4]. In most cases, segregation of an initially well-mixed granular mixture is undesirable, because it impacts the efficacy of later processes or the quality of final products. Therefore, understanding the mechanisms of granular segregation and developing efficient mixing technologies are of great importance in many industrial applications.

During the past several decades, the understanding of segregation in flowing granular materials has been advanced by extensive experimental, computational, and theoretical studies, examples include [5, 6, 7, 8, 9, 10, 11, 12, 13]. For size segregation in dense, sheared granular flows, a widely accepted percolation-based mechanism (sometimes called kinetic sieving [10]) posits that voids between particles are generated by shear and smaller particles are more likely than larger particles to fall into the voids under gravity. Consequently, large particles are pushed upward to the free surface, resulting in segregation in various flow geometries such as bounded heaps $[14,15,16]$, rotating tumblers $[17,18]$, and chutes $[19,20]$. Most of these studies have focused on segregation in steady granular flows with relatively simple kinematics and time invariant pattern formation. For example, for continuous quasi-2D bounded heap flow, when depositing size bidisperse mixtures onto the heap at a constant feed rate, smaller particles are deposited on the upstream portion of the heap, while larger particles flow to the downstream region of the heap, resulting in a segregated pattern in the streamwise direction $[14,15]$. As the particle size ratio increases, segregation becomes stronger, with a clear interface between the small and large particle enriched regions, which can cause inhomogeneity in subsequent processes such as hopper discharge or chute transfer. It is possible to minimize segregation by introducing counter-balancing mechanisms which include altering particle characteristics such as density $[6,7,13,21,22]$ and inelasticity [23], adding a small amount of liquid to dry particle mixtures making them more cohesive [24, 25, 26, 27, 28]

Email address: r-lueptow@northwestern.edu (Richard M. Lueptow)

Preprint submitted to Powder Technology

February 16, 2017

(C) 2017. This manuscript version is made available under the Elsevier user license

http://www.elsevier.com/open-access/userlicense/1.0/ 
or completely submerging the particles $[28,29,30]$. However, these methods are not always feasible in industrial applications, and in this work we focus on size segregation of dry spherical particles only.

Recent experimental [19] and theoretical [12] studies have shown that segregation strongly depends on details of the flow kinematics. As a result, segregation is likely to differ significantly between unsteady flows and steady flows. A handful of studies on unsteady monodisperse granular flows have revealed complicated kinematics during transient processes [31, 32, 33, 34, 35, 36]. For example, instantaneous velocity profiles measured during intermittent avalanches [34] exhibit a sharp increase and then a gradual decrease of the streamwise velocity during each avalanche. In rotating tumblers under a periodic forcing protocol [35], phase lags between the external forcing and the response of granular flow occur, indicating a delayed response of the granular system to external excitation. The more complicated kinematics in unsteady flows of monodisperse particles suggest increased challenges in understanding and predicting segregation in unsteady bidisperse flows, but also a richer range of possibilities for mixing or segregation-induced pattern formation $[18,37,38,39,40,41,42,43]$. An example is the dynamics of segregation pattern formation in timeperiodic tumbler flow [37,38]: discrete streaks of small particles form instead of the typical semicircular segregated core of small particles that occurs for steady flow, and the properties of the streaks depend on the modulation scheme.

Recent theoretical models have successfully captured the general structure of the patterns [44].

In this study, we explore the application of flow modulation to produce stratified layers in strongly segregating bidisperse mixtures with large size ratios by intentionally generating unsteady flows in a quasi-2D bounded heap. In bounded heap flows with constant feed rates, a stratified pattern of irregular, alternating layers can form spontaneously at low surface rise velocities due to intermittent avalanching $[14,45,46]$. At moderate surface rise velocities, the flow on the heap is steady and the stratified pattern transitions to the streamwise segregated pattern mentioned earlier. As the surface rise velocity is further increased, advection becomes the dominant transport mechanism and segregation is reduced $[14,12]$. However, a very high rise velocity is required to eliminate segregation of strongly segregating mixtures and is often hard to realize in practice since the rise velocity is inversely proportional to the cross-sectional area of the hopper [14]. In typical industrial settings, the rise velocity of bulk materials in a hopper often falls into the range where the streamwise segregated pattern occurs [14, 47, 48]. Although neither stratified nor streamwise segregated patterns are homogeneous, we will demonstrate that the stratified pattern represents the overall mixture when considered over the scale of several stratified layers. In addition, we show that, in comparison to the streamwise segregated pattern, the stratified pattern produced by feed flow modulation dramatically decreases the amount of segregation in hopper discharge flow due to layer remixing upon discharge. Therefore, using flow modulation to intentionally produce a stratified pattern when filling a hopper at industrially relevant operating conditions (e.g, at a feed rate where a streamwise segregated state otherwise occurs) could be valuable in many situations.

The remainder of the paper is organized as follows: section 2 describes the experimental setup; section 3 examines the flow-modulation-induced stratified patterns, proposes a mechanism for the flow-modulation-induced stratification, and explores the relation between the stratification properties and the modulation parameters; and section 4 presents the conclusions.

\section{Experimental methods}

The experimental apparatus (Fig. 1a) for the quasi-2D bounded heap flow consisted of a pair of parallel vertical rectangular plates - an aluminum back wall and a glass front wall for visualization. The vertical end walls and the base were aluminum. The width of the container was $W=0.69 \mathrm{~m}$, comparable to the flowing layer length in small industrial-scale silos. The gap between the front and back walls was $T=1.27 \mathrm{~cm}$. Further details regarding the experimental setup are described in Fan, et al. [14]. In this study, granular mixtures were dropped vertically into the gap between the front and back walls near the left bounding wall. Three mixtures of equal volumes of different-sized spherical glass particles were used: $0.50 \pm 0.04 \mathrm{~mm}$ and $1.69 \pm 0.05 \mathrm{~mm}$ (bidisperse); $0.50 \pm 0.04 \mathrm{~mm}$ and $2.00 \pm 0.07 \mathrm{~mm}$ (bidisperse); $1.00 \pm 0.05 \mathrm{~mm}, 2.00 \pm 0.07 \mathrm{~mm}$, and $2.98 \pm 0.06 \mathrm{~mm}$ (tridisperse). The material density of all particles was $2.59 \mathrm{~g} / \mathrm{cm}^{3}$.

An auger feeder (101-1-DD/2, Acrison, Inc., NJ, USA) controlled by an adjustable frequency AC drive (Powerflex 40, Rockwell Automation, Inc., IL, USA) fed particles from a height $0.65 \mathrm{~m}$ above the base onto the left end of the heap. Flow modulation was achieved by feeding the particles at two different $2 \mathrm{D}$ feed rates, $q=Q / T$, where $Q$ is the volumetric feed rate, see Fig. 1b. The flow was modulated between a fast phase at a higher feed rate $q_{f}$ for duration $t_{f}$ and a slow phase at a lower feed rate $q_{s}$ for duration $t_{s}$. The time for steady feed to be established was short (within 
$0.2 \mathrm{~s}$ ) compared to the duration of each phase (several seconds or more), suggesting its influence on the observed results was negligible.

To quantify stratification induced by flow modulation, videos were recorded during each experiment using a digital camera (D5300, Nikon Corporation, Tokyo, Japan). Figure 2a shows an image from an experiment demonstrating a stratified pattern achieved by flow modulation. In this study, we only examine stratification that occurs after the downstream end of the heap reaches the bounding wall (white box in Fig. 2a) [14]. To characterize the degree of stratification, the image of the boxed area was rotated by the angle of repose $\alpha$ and then sheared into a rectangular area with width $L$ corresponding to the length of the flowing layer (Fig. 2b). The uppermost layer of particles was not included in the analysis because it is influenced by the transient when the feed is stopped. Within this domain, we define a coordinate system where $x$ is the streamwise direction, $z$ is the depthwise direction, and the origin is at the lower left corner. Note that this domain includes the feed zone, which is typically $0.1 L$ wide in the cases tested. For each stratum (composed of a layer of large particles and a layer of small particles), $x_{L}$ denotes the leftmost extent of the large particle layer, $x_{R}$ denotes the rightmost extent of the small particle layer, and $\Delta x=x_{R}-x_{L}$ characterizes the interpenetration length, which quantifies the streamwise extent of stratification in the $x$-direction (Fig. 2b). Note that $x_{L}$ and $x_{R}$ were estimated visually for the set of strata in the image, which may introduce an error of as much as $\pm 1 \mathrm{~cm}$. These parameters are non-dimensionalized by $L: X_{L}=x_{L} / L, X_{R}=x_{R} / L$, and $\Delta X=\Delta x / L$. In addition, the pixel intensity of each image was averaged in the $x$-direction to produce an intensity profile in the $z$-direction (Fig. 2c). The wavelength of the intensity fluctuation, $\Delta H$, quantifies the thickness of pairs of strata in the $z$-direction [14, 49].

Particle Tracking Velocimetry (PTV) was used to determine the instantaneous velocity profiles near the transparent bounding sidewall of the apparatus. A high speed camera (FL3-U3-13Y3M-C, Point Grey Research Inc., Canada) recorded a series of images of the flowing layer at $400 \mathrm{frame} / \mathrm{s}$. An example of a close up image of the particles is shown in Fig. 2d. With proper lighting, the positions of the small and large particles were identified in each frame using a standard PTV code [50], and the instantaneous velocities of the particles were then calculated using particle positions from two consecutive frames. The streamwise velocity profiles in the $z$-direction at various locations along the flowing layer were then obtained using a spatial binning average [51]. The particles were grouped into $2 \mathrm{~mm}$ bins according to their depth in the $z$ direction, and an average of all particle velocities in each bin was calculated to obtain the velocity profiles. Combined with videos from the digital camera, PTV data was used to examine the mechanisms of stratification during modulated flow.

\section{Results and discussion}

\subsection{Comparing segregation with steady and modulated feed rates}

Fig. 3 compares the final segregation patterns for constant and modulated feed rates for two cases using $2 \mathrm{~mm}$ and $0.5 \mathrm{~mm}$ particles. The average feed rate for modulated flow, $\bar{q}=\left(q_{f} t_{f}+q_{s} t_{s}\right) /\left(t_{f}+t_{s}\right)$, is the same as the constant feed rate for each case. Two different average feed rates were investigated: a high feed rate, $\bar{q}=6.3 \mathrm{~cm}^{2} / \mathrm{s}$, corresponding to continuous flow for steady filling (Fig. 3a and b) and a low feed rate, $\bar{q}=1.5 \mathrm{~cm}^{2} / \mathrm{s}$, corresponding to discrete avalanching flow for steady filling (Fig. 3c and 3d) [15], noting that the transition feed rate from discrete avalanching flow to continuous flow occurs at about $q=4 \mathrm{~cm}^{2} / \mathrm{s}$. For continuous flow (cases with the higher $\bar{q}$ ), the constant feed rate produces a strong, streamwise-segregated pattern, in which small particles are concentrated in the upstream region and large particles are concentrated in the downstream region of the heap with a clear interface between them (Fig. 3a). As discussed in previous studies [14, 52], this segregated pattern occurs in continuous heap flow at moderate feed rates. In contrast, Fig. $3 \mathrm{~b}$ shows that a stratified pattern can be generated by using flow modulation at the same average feed rate as the constant feed rate used in Fig. 3a. In this case, the streamwise segregation still occurs with more small particles concentrated in the far upstream region and more large particles concentrated in the far downstream region. However, between these two regions, small and large particles form alternating layers parallel to the free surface. This pattern results in better overall, or effective, mixing normal to the heap surface than the fully streamwise-segregated pattern (Fig. 3a), as discussed earlier.

At the lower $\bar{q}$, stratified patterns occur with both constant and modulated feed rates but with different characteristics. For constant feed rate, spontaneous stratification occurs due to the interplay between particle segregation and intermittent avalanches [14] (Fig. 3c). Due to the random nature of the discrete avalanche flow, the stratified pattern formed at constant feed rate is nonuniform, evidenced by different interpenetration lengths and thicknesses of the 
strata. However, by adding a short period of a fast feed rate, a more uniform stratified pattern occurs at the same $\bar{q}$, as shown in Fig. 3d, where the interpenetration length and thickness of each stratum are nearly identical.

\subsection{Mechanisms for modulated-flow-induced stratification}

To demonstrate the mechanisms of modulated-flow-induced stratification, we focus on the formation of a single stratum in a modulated-flow experiment $\left(q_{f}=23.6 \mathrm{~cm}^{2} / \mathrm{s}, t_{f}=5 \mathrm{~s}, q_{s}=2.0 \mathrm{~cm}^{2} / \mathrm{s}\right.$, and $\left.t_{s}=20 \mathrm{~s}\right)$, shown in Fig. 4 . A single cycle of flow modulation (a fast phase and a slow phase) results in a single stratum. We track the stratum formation from the beginning of the fast phase, setting the elapsed time, $t$, to zero at this point, as shown in Fig. $1 \mathrm{~b}$. When the fast phase starts, a large volume of mixed particles falls onto the upstream end of the heap and flows downstream. As the materials flow downstream, particles segregate resulting in an excess of large particles at a "front", where they form a thick region, as shown in the white box in Fig. 4a. These large particles are followed upstream by the small particles in the rest of the flowing layer. This happens because in percolation-driven segregation, large particles rise to the free surface and small particles settle to the bottom of the flowing layer. The large particles near the surface move downstream faster than the small particles lower in the flowing layer $[15,34,35]$, as shown in the streamwise velocity profile at the approximate location of the front (Fig. 4b). This difference in streamwise velocity allows the large particles to flow further downstream relative to the small particles, thus forming the front. This observation is analogous to the experimental and theoretical results of Gray \& Kokelaar [53] and Gray \& Ancey [54] for discrete avalanches, where large particles are preferentially transported toward the front of a discrete avalanche. As the fast phase continues, the large particle enriched front thickens as more large particles from upstream flow to the front. This results in deposition of some large particles onto the heap as they flow down the heap. The small particles behind the large particle front flow over the deposited large particles, leaving a band of large particles beneath them (Fig. 4b). The location where large particles deposition begins is the left bound, $X_{L}$, of the stratum.

The small particle layer continues to flow downstream in the flowing layer after the feed rate switches to the slow phase. Fig. $4 \mathrm{c}$ shows the leading edge of the small particle layer flowing downstream during the slow feed rate phase at $x / L=0.55$ and $t=12 \mathrm{~s}$. This small particle enriched layer flows over the deposited large particle layer while simultaneously being covered by the large particles flowing down from upstream, similar to the 'small particle sandwich' described by Gray \& Kokelaar [53] and Gray \& Ancey [54]. As shown in Fig. 4d, some of the small particles penetrate into the voids between previously deposited or slowly flowing large particles at the bottom of the flowing layer, resulting in small scale remixing at the interface between the small particle layer and the deposited large particle layer. This phenomenon, referred to as spontaneous percolation, occurs in large size ratio mixtures $[10,20]$, but it does not adversely affect the stratification in the experiments conducted. Note that the thickness of the flowing layer is only about $8 \mathrm{~mm}$ in Fig. 4d, so the large particles remain deposited beneath the flowing layer and are not carried further downstream by the flow. The flowing small particles are eventually buried by the flowing large particles above them, resulting in the small particles being deposited below the flowing layer. The position where the leading edge of the layer of small particles stops is $X_{R}$. For a slow phase with lower $q_{s}$, the leading edge of the small particle layer travels to a position further downstream because it remains in the flowing layer for a longer time, resulting in larger $X_{R}$. For a higher $q_{s}$, the leading edge of the small particle layer stops further upstream due to its shorter residence time in the flowing layer, leading to a smaller $X_{R}$. Note that the slow feed rate in the modulated cases sometimes falls into the range where intermittent flow occurs for a steady feed rate [14, 55]. However, this does not seem to influence the uniformity of the stratified layers, because the flow in the transition from fast to slow feed rate is continuous.

In general, the fast phase results in a quick segregation of the particles in the upstream portion of the heap, where the large particles in a large particle enriched front are deposited as the front travels downstream, and small particles flow over these deposited large particles, initiating new layers of small and large particles along the streamwise direction. The slow phase allows the small particle layer to extend further downstream. The mechanism for the formation of the stratified layers in modulated flow is different from the mechanism for stratification of mixtures of different size and shape particles with a constant feed rate $[45,56,57]$. In the latter case, a difference in particle surface roughness or shape is necessary, and the stratification depends on the formation of kinks traveling upstream from the end wall [57]. The mechanism described here is more similar to that for the spontaneous stratification of different sized spherical particles with a similar repose angles (Fig. 3c) [14]. Under low steady feed rates, the flow is intermittent as the flowing layer "freezes" from time to time [14,55]. And after the free surface ceases to flow, the new particles fed onto the heap accumulate near the feed zone, and soon starts to flow downstream again, possibly because 
the local maximum static angle [58] is reached due to the accumulation. This process plays a similar role as the fast phase does with modulated flow in terms of accumulating newly deposited particles far upstream. As these particles flow downstream, segregation occurs resulting in a large particle front where some of the large particles are deposited and then covered by a small particle layer that travels downstream, similar to the slow phase in the modulated cases, until the flowing layer freezes again and the formation of next layer starts. However, in spontaneous stratification, the formation of the stratified layers is less uniform due to the randomness in the "freezing" of the flowing layer [55], while the controlled modulation of the feed rate results in stratified layers that are more uniform. More importantly from a practical standpoint, the modulated-flow-induced stratification can occur at higher average feed rates.

\subsection{A parametric study}

To study how the feed modulation parameters control stratification, we conducted 79 experiments using an equal volume mixture of $1.7 \mathrm{~mm}$ and $0.5 \mathrm{~mm}$ particles, and measured $\Delta H, X_{L}, \Delta X$, and $X_{R}$ for each experiment to quantify stratification under various flow conditions. The $2 \mathrm{D}$ feed rate ranged from $0.6 \mathrm{~cm}^{2} / \mathrm{s}$ to $33.9 \mathrm{~cm}^{2} / \mathrm{s}$ and the phase durations varied from $2 \mathrm{~s}$ to $60 \mathrm{~s}$. In each case, the stratification parameters $\left(\Delta H, X_{L}, \Delta X\right.$, and $\left.X_{R}\right)$ were calculated by averaging over all strata that formed after the heap reached the downstream end of the apparatus (the white box in Fig. 2a and 2b).

The thickness of each stratum $\Delta H$ can be predicted from the total volume of particles in each feed cycle, $V_{t o t}=$ $\left(q_{f} t_{f}+q_{s} t_{s}\right) T$, assuming that each feed cycle results in a single layer of each particle size. The relation between $\Delta H$ and $V_{\text {tot }}$ is

$$
\Delta H=\frac{V_{t o t} \cos \alpha}{W T},
$$

where $\alpha$ is the dynamic repose angle of the mixture, which in the cases tested here ranged from approximately $27^{\circ}$ to $33^{\circ}$ depending on the feed rate and consistent with previous results [14]. Here, for simplicity, we use an average value of $\alpha=30^{\circ}$ for all feed rates. To confirm the relation in Eq. (1), experimental values of $\Delta H$ normalized by the nominal mean particle diameter $\bar{d}=\left(d_{l}+d_{s}\right) / 2=1.1 \mathrm{~mm}$ are plotted as function of the right hand side of Eq. (1), also normalized by $\bar{d}$. The experimental data match Eq. (1) for $V_{t o t} \cos \alpha / W T \bar{d}$ above a critical value around 10 (equivalent to $V_{\text {tot }}=110 \mathrm{~cm}^{3}$ ), corresponding to a minimum stratum thickness of approximately $10 \bar{d}$, which is approximately the flowing layer thickness shown in Fig. 4. A stratum thinner than the flowing layer thickness, which is typically $5 \bar{d}$ to $10 \bar{d}$, is unlikely to form since the stratum will be entrained into the flowing layer in the subsequent feed cycle and combine with the new stratum. Therefore, for low $V_{t o t}$, particles from more than one feed cycle end up merging into a single stratum, similar to the merging of streaks in modulated circular tumbler flow [38]. As a result, $\Delta H$ is greater than the value predicted by Eq. (1) for small $V_{t o t}$.

As discussed in section 3.2, the left bound of the strata, $X_{L}$, indicates where small particles flow over previously deposited large particles. These large particles are from either the previous slow phase or the large particle front during the fast phase. In both situations, a lower feed rate can deposit large particles closer to the feed zone (smaller $X_{L}$ ), because the streamwise segregation is generally stronger at a lower feed rate [14]. This suggests that the average feed rate $\bar{q}$ is the dominant factor determining $X_{L}$. Figure $5 \mathrm{~b}$ confirms this conjecture within the ranges of parameters tested by showing a linear relation between $X_{L}$ and the nondimensional feed rate $\tilde{q}=\bar{q} / \sqrt{g \bar{d}^{3}}$, where $g=9.81 \mathrm{~m} / \mathrm{s}^{2}$ is the gravitational acceleration. The nondimensionalization by $\sqrt{g \bar{d}^{3}}$ reflects a flux scale which is the product of a length scale $\bar{d}$ and a commonly used velocity scale $\sqrt{g} \bar{d}$, similar to scalings used previously [15, 32, 52]. $X_{L}$ cannot be smaller than the width of the feed zone, which is about $0.1 \mathrm{~L}$ in this study, as indicated by the dashed line in Fig. $5 \mathrm{~b}$.

The interpenetration depth $\Delta X$, depends on the maximum distance that the small particle layer travels in the streamwise direction, which is determined by both the time small particles remain in the flowing layer and the speed at which small particles travel down the heap before being deposited. Since most of the small particle layer motion occurs during the slow phase, the slow phase time duration, $t_{s}$, and feed rate, $q_{s}$, are likely the dominant factors determining $\Delta X$. Longer $t_{s}$ allows more time for the small particle layer to grow, while larger $q_{s}$ results in a faster rise velocity of the heap surface during the slow phase $\left(v_{r}=q_{s} / W\right)$, which causes small particles to be buried faster by large particles as they travel down the heap. Fig. $5 \mathrm{c}$ shows a correlation between the non-dimensional interpenetration depth and a non-dimensional parameter that includes both modulation parameters, $\tilde{t}_{s} \tilde{q}_{s}^{-2}$, where $\tilde{t}_{s}=t_{s} / \sqrt{\bar{d} / g}$ and $\tilde{q}_{s}=q_{s} / \sqrt{g \bar{d}^{3}}$, where $\sqrt{g \bar{d}}$ is the timescale for a particle to fall a particle diameter [32]. The data clearly show that when $t_{s}$ increases or $q_{s}$ decreases, $\Delta X$ can increase up to 0.8 , which means strata span nearly the entire length of the 
slope. Note that the physics underlying the logarithmic relation between $\Delta X$ and $\tilde{t}_{s} \tilde{q}_{s}^{-2}$ in Fig. $5 \mathrm{c}$ is unclear and needs further investigation. In addition, there are several secondary effects that can influence $\Delta X$ including the fast phase flow parameters, the difference in the repose angle of the two phases, and the curved free surface that occurs at high feed rates [59], perhaps accounting for the data scatter in Fig. 5c. Based on the correlations from Fig. 5b and 5c, the right bound of the strata, $X_{R}=X_{L}+\Delta X$, can be relatively accurately predicted, as shown in Fig. $5 \mathrm{~d}$.

To this point, we have demonstrated that it is possible to generate a wide range of stratified patterns with modulation of the heap feed rate. We now quantify the degree of inhomogeneity in the streamwise direction for these patterns using the Danckwerts intensity of segregation [60]:

$$
I_{d}(c)=\frac{1}{\bar{c}(1-\bar{c})} \int_{0}^{L}[c(x)-\bar{c}]^{2} d x
$$

where, $\bar{c}=(1 / L) \int_{0}^{L} c(x) d x$ is the overall spatially averaged concentration for each species, and $c(x)$ is the local species concentration. The intensity of segregation is a measure of the deviation of local concentration from the overall average, and it ranges from $I_{d}=0$, which indicates perfect mixing and corresponds to the strata extending the full length of the flowing layer, to $I_{d}=1$, which indicates complete segregation corresponding to no strata at all, but instead a region of pure small particles in the upstream portion of the heap and pure large particles in the downstream portion. The concentration, $c$, is measured using the pixel intensity of processed images (Fig. 2) by assuming a linear relation between the intensity and particle concentration at the wall [14]. The heap is divided into 20 equal-sized nonoverlapping bins (each bin is $4 \mathrm{~cm}$ wide) in the streamwise direction $x$, and the local concentration is averaged over the entire depth ( $z$-direction). Figure 6 demonstrates how the intensity of segregation decreases as the interpenetration depth $\Delta X$ increases, indicating that $\Delta X$ is a reasonable proxy for the degree of mixing in the streamwise direction. For $\Delta X$ less than $0.2, I_{d}$ reaches its maximum value of about 0.8 , which is equivalent to $I_{d}$ for a completely streamwise segregated pattern at a constant feed rate around $q=25 \mathrm{~cm}^{2} / \mathrm{s}$. When $\Delta X$ is larger than $0.2, I_{d}$ decreases linearly towards 0 . In the cases tested, $I_{d}$ never reaches 0 and $\Delta X$ never reaches 1 , because mixed material is always present in the feed zone and a small region of large particles persists at the downstream end of the flowing layer.

The measured degree of mixing in the $z$-direction depends on the averaging length scale. If the bin size is smaller than $\Delta H$ in the $\mathrm{z}$ direction, the degree of mixing will be small. Thus, the metric for mixing in the $z$-direction will depend on the specific application. For example, as shown in the next section, the small scale inhomogeneity in the $z$-direction is immaterial during hopper discharge, because the discharge flow effectively remixes the particle layers as they exit the hopper.

\subsection{Potential application in hopper discharge}

In this section, we demonstrate the potential application of modulated-flow-induced stratification to improve mixture homogeneity during hopper discharge. To do so, the apparatus was slightly modified into a quasi-2D onesided funnel flow hopper by inclining the bottom base and opening an outlet at the left end, see Fig. 7. Three different initial states were created by filling a size bidisperse mixture $(0.5 \mathrm{~mm}$ and $2.0 \mathrm{~mm}$ particles) at the left end while the outlet was closed; a streamwise segregated pattern was obtained by filling the hopper at a constant feed rate, $q=6.0 \mathrm{~cm}^{2} / \mathrm{s}$ and letting particles naturally segregate [14]; a stratified initial condition was obtained by filling the hopper using a modulated feed rate with $q_{f}=40.0 \mathrm{~cm}^{2} / \mathrm{s}, t_{f}=2.5 \mathrm{~s}, q_{s}=2.0 \mathrm{~cm}^{2} / \mathrm{s}$, and $t_{s}=23 \mathrm{~s}$; and a completely stratified pattern was artificially generated by manually filling alternately with large and small particles.

To characterize the degree of mixing in the discharge, the outflow from the hopper was divided into 5 equalvolume samples sequentially, and the small particle concentration, $c_{s}$, in each sample was measured. An average of three identical experiments were used for each initial condition, and the results are shown in Fig. $7 \mathrm{~d}$. In all cases, the particles on the left side of the hopper are the first to discharge. For the discharge of the initially streamwise segregated pattern (Fig. 7a right), the small particles concentrated in the left (upstream) region are discharged first followed by the large particles in the right (downstream) region, resulting in a significant decrease in the small particle concentration as hopper discharge progresses. For the discharge of the initially stratified pattern induced by modulated-flow (Fig. $7 \mathrm{~b}$ right), both the small and large particle layers contribute to the outflow simultaneously, resulting in remixing. This leads to an increase of homogeneity in the discharge compared to the streamwise segregated pattern, although segregation still exists because the stratified layers do not extend the entire width of the hopper. When discharging materials from the initially completely stratified pattern (Fig. 7c right), which can be considered as an optimized 
version of the modulated-flow-induced stratified layers in Fig. 7b, all the stratified layers contribute to the outflow simultaneously during the entire discharge process, leading to almost perfect mixing in the outflow (Fig. 7d). Moreover, this homogeneity created by the stratified layers is independent of the sample size because the small and large particles contribute equally to the outflow at any instant. These results demonstrate the potential of applying flow modulation to increase mixture homogeneity during hopper discharge. Of course, more work is needed to optimize modulated-flow-induced stratification.

\subsection{Tridisperse mixtures}

Up to this point, we have shown the potential of flow-modulation-induced stratification for size bidisperse mixtures. The underlying mechanism does not limit the application to bidisperse mixtures. Fig. 8 shows a comparison between steady and modulated bounded heap flow at comparable average feed rates for a tridisperese granular mixture of equal volumes of $1 \mathrm{~mm}, 2 \mathrm{~mm}$, and $3 \mathrm{~mm}$ glass particles. For the steady feed rate (Fig. 8a), the three species generally segregate in the streamwise direction, with small particles depositing upstream, large particles depositing downstream, and medium-sized particles depositing in the middle of the heap, consistent with previous results [61], though there is some minor stratification due to the low feed rate. For a modulated feed rate, stratification occurs in the same fashion as for bidisperse mixtures, forming distinct alternating layers, as shown in Fig. 8b. In the close-up image of the stratified region, ordered layers of small, medium, and large particles are clearly visible, and each species is in contact with the other two species. Although the global mixing is imperfect, the modulated stratified pattern has better mixing than the steady flow pattern, especially for small and large particles. In the steady flow pattern, large and small particles are completely separated by the medium-sized particle region, while in the modulated flow pattern they are interspersed with each other at the layer interfaces. Moreover, the medium-sized particles are more dispersed along the streamwise direction in the modulated flow pattern.

\section{Conclusions}

In this paper, we have shown that flow modulation realized by alternating between high and low feed rates can generate stratified patterns of size disperse granular mixtures in a quasi-2D bounded heap. During the fast phase, large particles are deposited in the upstream portion of the heap behind the large particle front and then covered by the small particles flowing from upstream to initiate the stratified layers, while during the slow phase, the small particle layer travels downstream until it is covered by large particles. We have quantified the relationships between the stratification patterns and the modulation parameters based on this mechanism. These relations indicate how to achieve stratified patterns rather than streamwise segregated patterns at the same average feed rate in heap flow by properly selecting the modulation parameters. Based on the Danckwerts intensity of segregation and a preliminary hopper discharge study, we demonstrated that the flow-modulated stratified pattern can reduce the overall inhomogeneity in a heap leading to better mixing during hopper discharge, which has great potential to limit particle segregation in many industrial processes.

Stratification via flow modulation occurs in tridisperse size mixtures and should also occur for mixtures of particles differing in material density, because density segregation in bounded heaps shares many similarities with size segregation [51] and layering of density bidisperse materials has been observed in rotating tumbler flow [62]. Further work is necessary to better explore these topics. Meanwhile, a continuum-based theoretical framework capable of predicting segregation at constant feed rates $[12,44,52]$ can potentially be adapted to predict flow-modulated stratification and further optimize this approach. This work is currently underway.

\section{Acknowledgments}

We gratefully acknowledge the financial support of The Dow Chemical Company and the National Science Foundation, Grant No. CBET-1511450. 


\section{References}

\section{References}

[1] J. Bridgwater, Mixing of powders and granular materials by mechanical means-A perspective, Particuology 10 (4) (2012) $397-427$.

[2] J. Ottino, D. Khakhar, Mixing and segregation of granular materials, Annu. Rev. Fluid Mech. 32 (1) (2000) 55-91.

[3] F. J. Muzzio, T. Shinbrot, B. J. Glasser, Powder technology in the pharmaceutical industry: the need to catch up fast, Powder Technol. 124 (1) (2002) $1-7$.

[4] K. Hill, A. Caprihan, J. Kakalios, Bulk segregation in rotated granular material measured by magnetic resonance imaging, Phys. Rev. Lett. 78 (1) (1997) 50.

[5] J. Drahun, J. Bridgwater, The mechanisms of free surface segregation, Powder Technol. 36 (1) (1983) 39-53.

[6] N. Jain, J. M. Ottino, R. M. Lueptow, Regimes of segregation and mixing in combined size and density granular systems: an experimental study, Granul. Matter 7 (2-3) (2005) 69-81.

[7] G. Félix, N. Thomas, Evidence of two effects in the size segregation process in dry granular media, Phys. Rev. E 70 (5) (2004) 051307.

[8] D. Rapaport, Simulational studies of axial granular segregation in a rotating cylinder, Phys. Rev. E 65 (6) (2002) 061306.

[9] A. Tripathi, D. Khakhar, Density difference-driven segregation in a dense granular flow, J. Fluid Mech. 717 (2013) 643-669.

[10] S. Savage, C. Lun, Particle size segregation in inclined chute flow of dry cohesionless granular solids, J. Fluid Mech. 189 (1988) $311-335$.

[11] J. Gray, A. Thornton, A theory for particle size segregation in shallow granular free-surface flows, Proc. R. Soc. A 461 (2057) (2005) 1447-1473.

[12] Y. Fan, C. P. Schlick, P. B. Umbanhowar, J. M. Ottino, R. M. Lueptow, Modelling size segregation of granular materials: the roles of segregation, advection and diffusion, J. Fluid Mech. 741 (2014) 252-279.

[13] M. Larcher, J. T. Jenkins, The evolution of segregation in dense inclined flows of binary mixtures of spheres, J. Fluid Mech. 782 (2015) 405-429.

[14] Y. Fan, Y. Boukerkour, T. Blanc, P. B. Umbanhowar, J. M. Ottino, R. M. Lueptow, Stratification, segregation, and mixing of granular materials in quasi-two-dimensional bounded heaps, Phys. Rev. E 86 (5) (2012) 051305.

[15] Y. Fan, P. B. Umbanhowar, J. M. Ottino, R. M. Lueptow, Kinematics of monodisperse and bidisperse granular flows in quasi-two-dimensional bounded heaps, Proc. R. Soc. A 469 (2013) 20130235.

[16] S. H. Gharat, D. Khakhar, Granular segregation in quasi-2d rectangular bin, in: POWDERS AND GRAINS 2013: Proceedings of the 7th International Conference on Micromechanics of Granular Media, Vol. 1542, AIP Publishing, 2013, pp. 755-758.

[17] N. Jain, J. M. Ottino, R. M. Lueptow, Combined size and density segregation and mixing in noncircular tumblers, Phys. Rev. E 71 (5) (2005) 051301.

[18] J. Gray, K. Hutter, Pattern formation in granular avalanches, Contin. Mech. Thermodyn. 9 (6) (1997) 341-345.

[19] S. Wiederseiner, N. Andreini, G. Épely-Chauvin, G. Moser, M. Monnereau, J. Gray, C. Ancey, Experimental investigation into segregating granular flows down chutes, Phys. Fluids 23 (1) (2011) 013301.

[20] A. Thornton, T. Weinhart, S. Luding, O. Bokhove, Modeling of particle size segregation: calibration using the discrete particle method, Int. J. Mod. Phys. C 23 (08) (2012) 1240014.

[21] D. Tunuguntla, O. Bokhove, A. Thornton, A mixture theory for size and density segregation in shallow granular free-surface flows, J. Fluid. Mech. 749 (2014) 99-112.

[22] J. Gray, C. Ancey, Particle-size and-density segregation in granular free-surface flows, J. Fluid Mech. 779 (2015) $622-668$.

[23] R. Brito, H. Enríquez, S. Godoy, R. Soto, Segregation induced by inelasticity in a vibrofluidized granular mixture, Phys. Rev. E 77 (6) (2008) 061301.

[24] H. Li, J. McCarthy, Controlling cohesive particle mixing and segregation, Phys. Rev. Lett. 90 (18) (2003) 184301.

[25] A. Samadani, A. Kudrolli, Segregation transitions in wet granular matter, Phys. Rev. Lett. 85 (24) (2000) 5102.

[26] C. C. Liao, S. S. Hsiau, T. H. Tsai, C. H. Tai, Segregation to mixing in wet granular matter under vibration, Chem. Eng. Sci. 65 (3) (2010) 1109-1116.

[27] P. Liu, R. Yang, A. Yu, The effect of liquids on radial segregation of granular mixtures in rotating drums, Granul. Matter 15 (4) (2013) $427-436$.

[28] A. Samadani, A. Kudrolli, Angle of repose and segregation in cohesive granular matter, Physical Review E 64 (5) (2001) 051301.

[29] J. W. Vallance, S. B. Savage, Particle segregation in granular flows down chutes, in: IUTAM Symposium on Segregation in Granular flows, Springer, 2000, pp. 31-51.

[30] A. R. Thornton, J. Gray, A. Hogg, A three-phase mixture theory for particle size segregation in shallow granular free-surface flows, Journal of Fluid Mechanics 550 (2006) 1

[31] R. Spurling, J. Davidson, D. Scott, The transient response of granular flows in an inclined rotating cylinder, Chem. Eng. Res. Des. 79 (1) (2001) 51-61.

[32] P. Jop, Y. Forterre, O. Pouliquen, Initiation of granular surface flows in a narrow channel, Phys. Fluids 19 (8) (2007) 088102.

[33] N. A. Pohlman, D. F. Paprocki Jr, Transient behavior of granular materials as result of tumbler shape and orientation effects, Granul. Matter 15 (1) (2013) 39-47.

[34] S. C. du Pont, R. Fischer, P. Gondret, B. Perrin, M. Rabaud, Instantaneous velocity profiles during granular avalanches, Phys. Rev. Lett. 94 (4) (2005) 048003.

[35] N. A. Pohlman, J. M. Ottino, R. M. Lueptow, Unsteady granular flows in a rotating tumbler, Phys. Rev. E 80 (3) (2009) 031302.

[36] S. Parez, E. Aharonov, R. Toussaint, Unsteady granular flows down an inclined plane, Phys. Rev. E 93 (4) (2016) 042902.

[37] S. Fiedor, J. Ottino, Mixing and segregation of granular matter: multi-lobe formation in time-periodic flows, J. Fluid Mech. 533 (2005) 223-236.

[38] R. D. East, P. McGuinness, F. Box, T. Mullin, I. Zuriguel, Granular segregation in a thin drum rotating with periodic modulation, Phys. Rev. E 90 (5) (2014) 052205. 
[39] D. Shi, A. A. Abatan, W. L. Vargas, J. McCarthy, Eliminating segregation in free-surface flows of particles, Phys. Rev. Lett. 99 (14) (2007) 148001.

[40] J. Fineberg, From cinderella's dilemma to rock slides, Nature 386 (1997) 323-324.

[41] O. Pouliquen, J. Delour, S. B. Savage, Fingering in granular flows, Nature 386 (1997) 816-817.

[42] D. R. Tunuguntla, T. Weinhart, A. R. Thornton, Comparing and contrasting size-based particle segregation models, Comput. Part. Mech. (2016) $1-19$.

[43] A. Edwards, N. Vriend, Size segregation in a granular bore, Phys. Rev. Fluids 1 (6) (2016) 064201.

[44] C. P. Schlick, Y. Fan, P. B. Umbanhowar, J. M. Ottino, R. M. Lueptow, Granular segregation in circular tumblers: theoretical model and scaling laws, J. Fluid Mech. 765 (2015) 632-652.

[45] H. A. Makse, S. Havlin, P. R. King, S. Eugene, Spontaneous stratification in granular mixtures, Nature 386 (1997) 379-382.

[46] J. Baxter, U. Tüzün, D. Heyes, I. Hayati, P. Fredlund, Stratification in poured granular heaps, Nature 391 (6663) (1998) $136-136$.

[47] K. Shinohara, K. Shoji, T. Tanaka, Mechanism of size segregation of particles in filling a hopper, Ind. Eng. Chem. Proc. Des. Dev. 11 (3) (1972) 369-376.

[48] N. Standish, Studies of size segregation in filling and emptying a hopper, Powder Technol. 45 (1) (1985) $43-56$.

[49] Y. Grasselli, H. Herrmann, Experimental study of granular stratification, Granul. Matter 1 (1) (1998) 43-47.

[50] J. C. Crocker, D. G. Grier, Methods of digital video microscopy for colloidal studies, J. Colloid Interface Sci. 179 (1) (1996) $298-310$.

[51] H. Xiao, P. B. Umbanhowar, J. M. Ottino, R. M. Lueptow, Modelling density segregation in flowing bidisperse granular materials, in: Proc. R. Soc. A, Vol. 472, The Royal Society, 2016, p. 20150856.

[52] C. P. Schlick, Y. Fan, A. B. Isner, P. B. Umbanhowar, J. M. Ottino, R. M. Lueptow, Modeling segregation of bidisperse granular materials using physical control parameters in the quasi-2d bounded heap, AIChE J. 61 (5) (2015) 1524-1534.

[53] J. Gray, B. Kokelaar, Large particle segregation, transport and accumulation in granular free-surface flows, J .Fluid Mech. 652 (2010) 105137.

[54] J. Gray, C. Ancey, Segregation, recirculation and deposition of coarse particles near two-dimensional avalanche fronts, J. Fluid Mech. 629 (2009) 387-423.

[55] A. R. Abate, H. Katsuragi, D. J. Durian, Avalanche statistics and time-resolved grain dynamics for a driven heap, Phys. Rev. E 76 (6) (2007) 061301.

[56] H. A. Makse, Stratification instability in granular flows, Phys. Rev. E 56 (6) (1997) 7008.

[57] H. A. Makse, R. C. Ball, H. E. Stanley, S. Warr, Dynamics of granular stratification, Phys. Rev. E 58 (3) (1998) 3357.

[58] Y. Grasselli, H. Herrmann, On the angles of dry granular heaps, Physica A 246 (3) (1997) 301-312.

[59] Y. Fan, K. Jacob, B. Freireich, R. M. Lueptow, Segregation of granular materials in bounded heap flow: a review, Powder Technol. Under review.

[60] P. Danckwerts, The definition and measurement of some characteristics of mixtures, Appl. Sci. Res. A 3 (4) (1952) $279-296$.

[61] C. Schlick, A. Isner, B. Freireich, Y. Fan, P. Umbanhowar, J. Ottino, R. Lueptow, A continuum approach for predicting segregation in flowing polydisperse granular materials, J. Fluid Mech. 797 (2016) 95-109.

[62] C. C. Liao, S. S. Hsiau, H. C. Nien, Effects of density ratio, rotation speed, and fill level on density-induced granular streak segregation in a rotating drum, Powder Technol. 284 (2015) 514-520. 
(a)

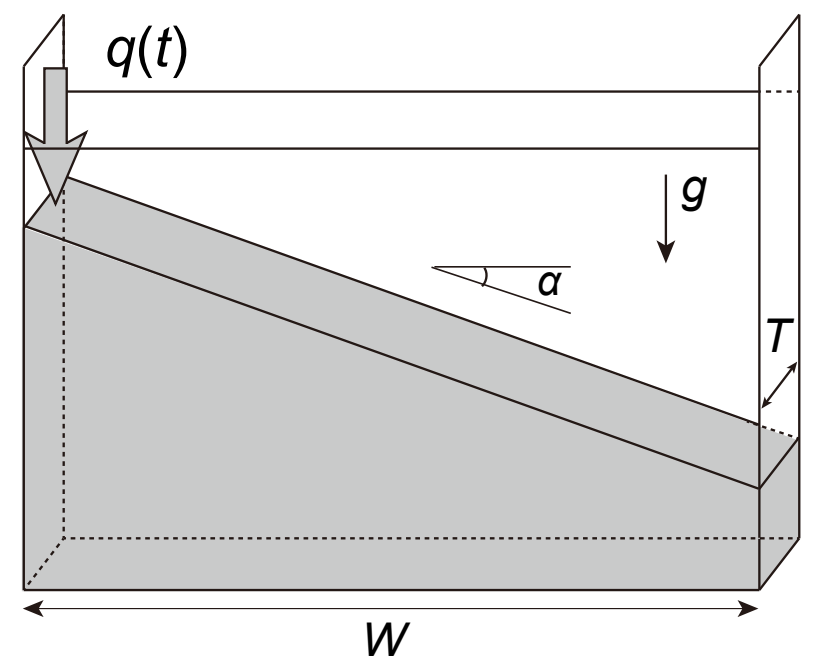

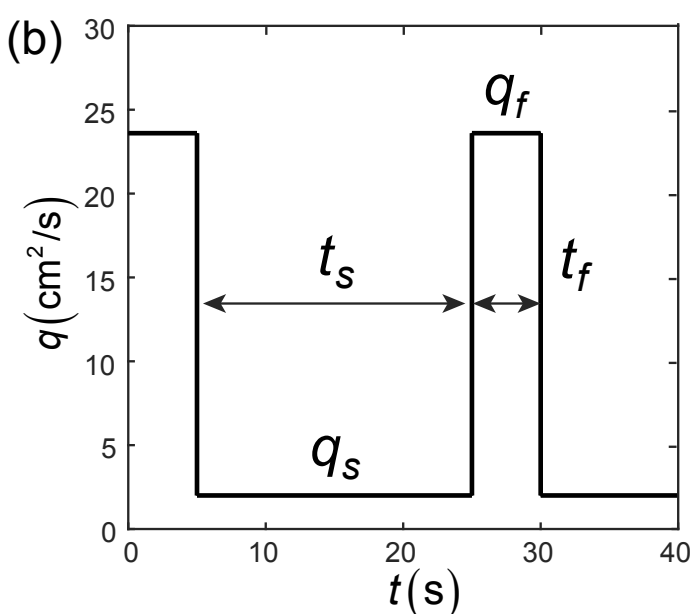

Figure 1: (a) Sketch of a quasi-2D bounded heap (not to scale) with width $W$ and thickness $T$. Size bidisperse mixtures are fed onto the heap near the left end at modulated feed rate $q(t)$. (b) Feed rate modulation scheme for $q_{f}=23.6 \mathrm{~cm}^{2} / \mathrm{s}, t_{f}=5 \mathrm{~s}, q_{s}=2.0 \mathrm{~cm}^{2} / \mathrm{s}$, and $t_{s}=20 \mathrm{~s}$. 
(a)

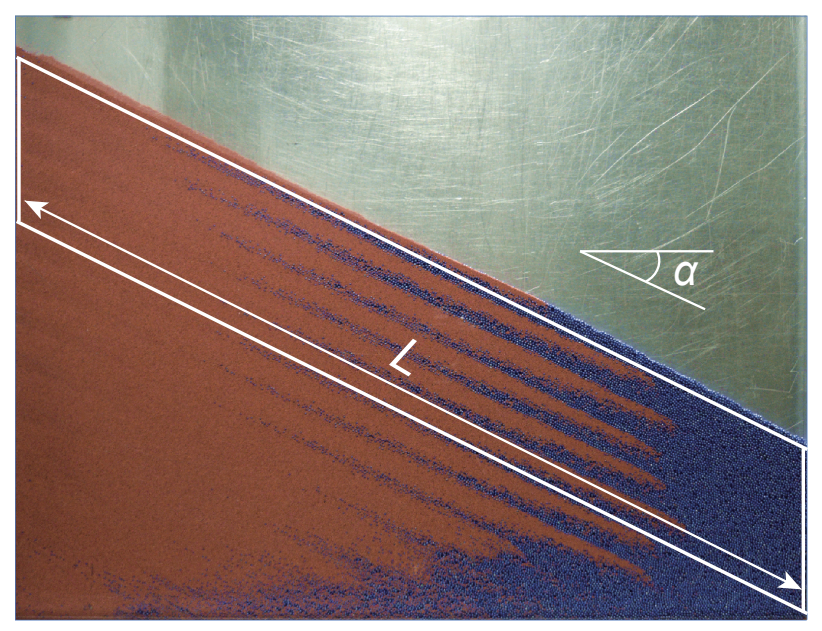

(b)

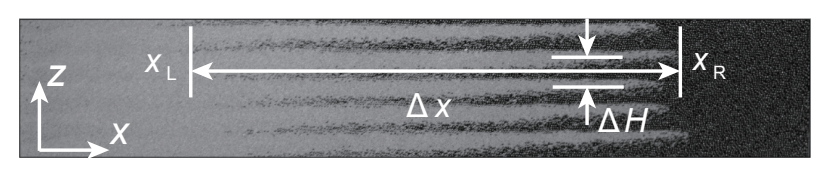

(c)

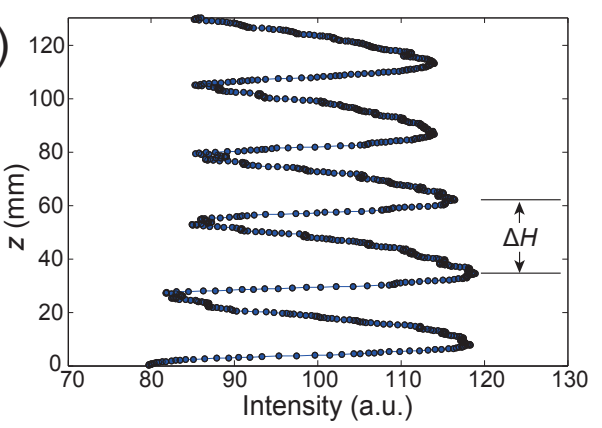

(d)

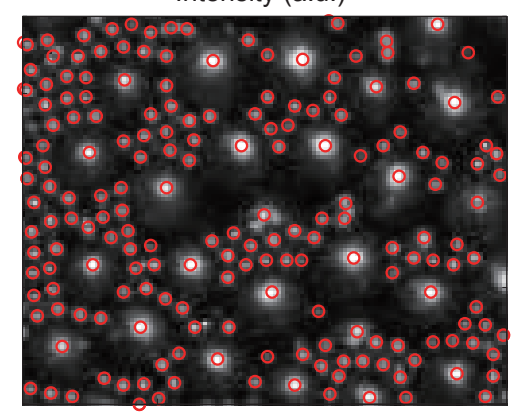

Figure 2: Analysis methods for experimental results. (a) An example of the final image in an experiment with $2 \mathrm{~mm}$ (blue) and $0.5 \mathrm{~mm}$ (red) glass spherical particles. The boxed area represents the pattern formed after the heap has reached its full horizontal extent. (b) Shearing the boxed area into a rectangular domain. (c) Streamwise-averaged image intensity vs. depth. (d) An example of particle identification in particle tracking velocimetry (identified particles are marked with a red circle). 

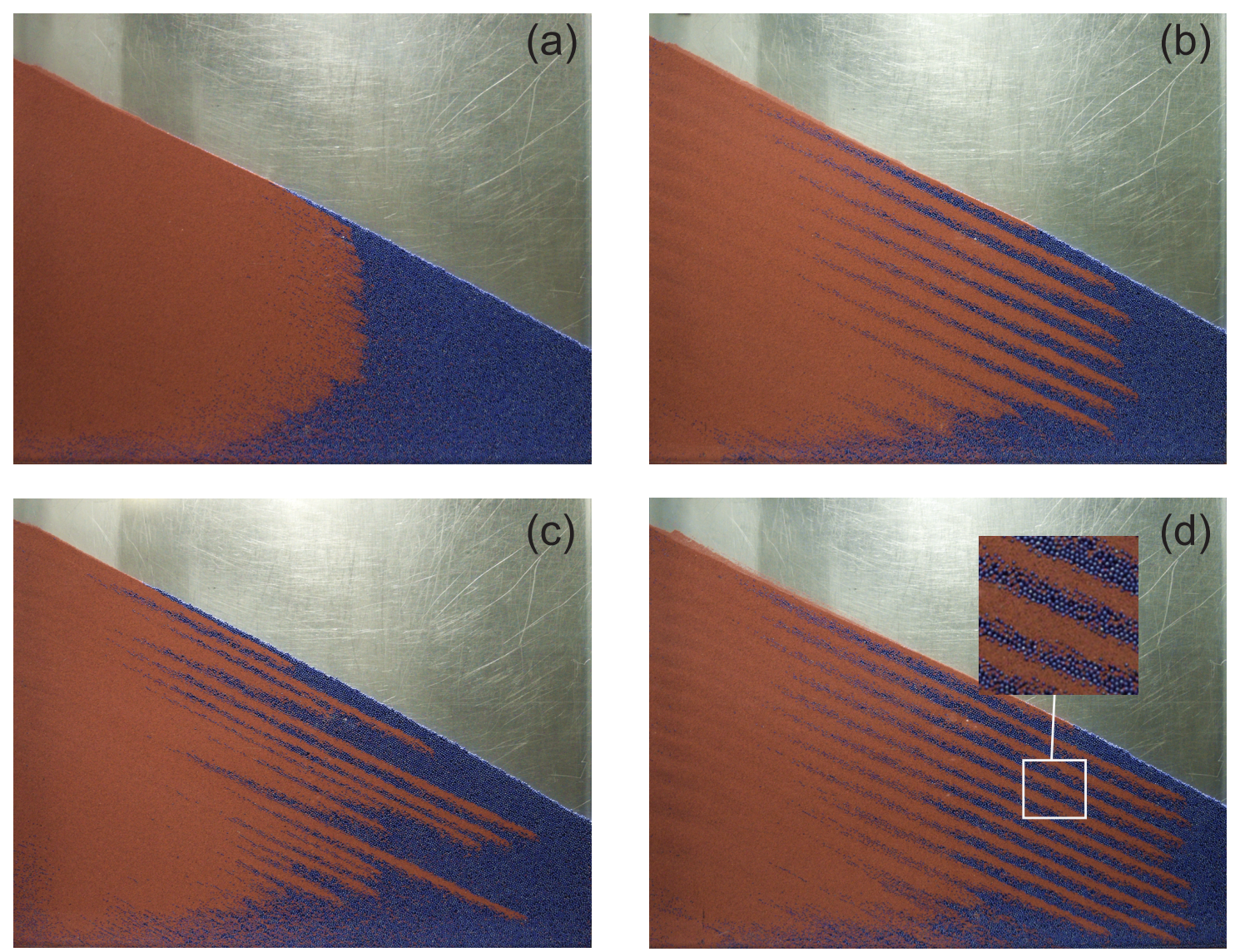

Figure 3: Patterns generated from steady (left column) and modulated (right column) filling using $2 \mathrm{~mm}$ (blue) and $0.5 \mathrm{~mm}$ (red) particles. (a) Uniform streamwise segregation with constant feed rate $q=6.3 \mathrm{~cm}^{2} / \mathrm{s}$. (b) Uniform stratification with modulated flow at the same average flow rate as in (a), using $q_{f}=23.6 \mathrm{~cm}^{2} / \mathrm{s}, t_{f}=5 \mathrm{~s}, q_{s}=$ $2.0 \mathrm{~cm}^{2} / \mathrm{s}$, and $t_{s}=20 \mathrm{~s}$. (c) Heterogeneous stratification in avalanching regime with constant $q=1.5 \mathrm{~cm}^{2} / \mathrm{s}$. $(\mathrm{d})$ Uniform stratification with modulated flow at same average flow rate as in (c), using $q_{f}=31.0 \mathrm{~cm}^{2} / \mathrm{s}, t_{f}=3 \mathrm{~s}$, $q_{s}=0.3 \mathrm{~cm}^{2} / \mathrm{s}$, and $t_{s}=77 \mathrm{~s}$. 

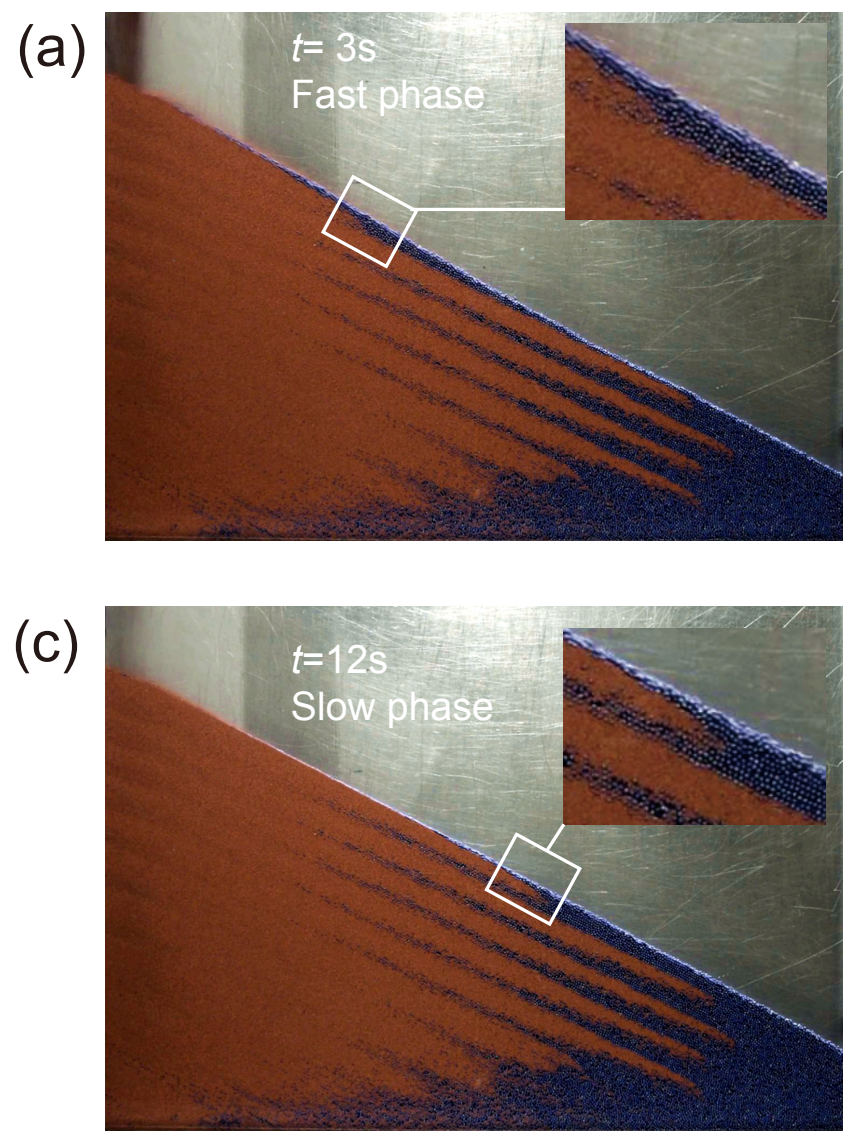

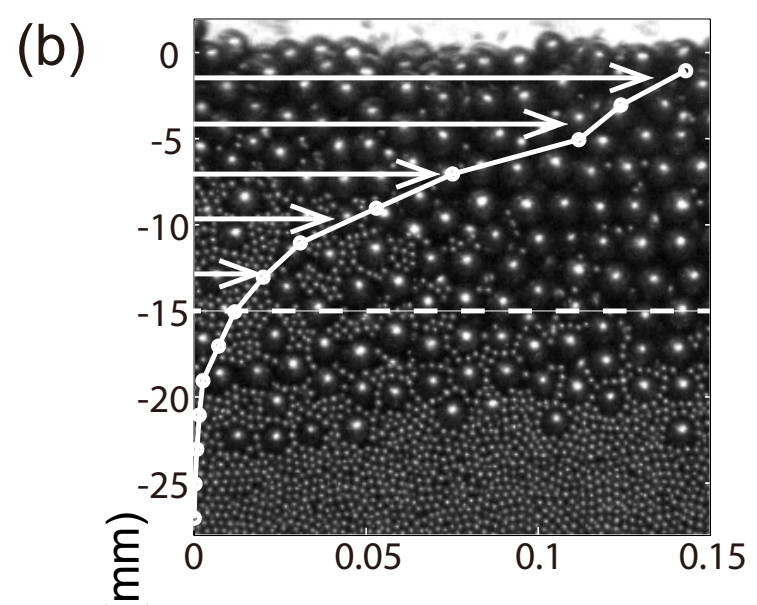

(d)

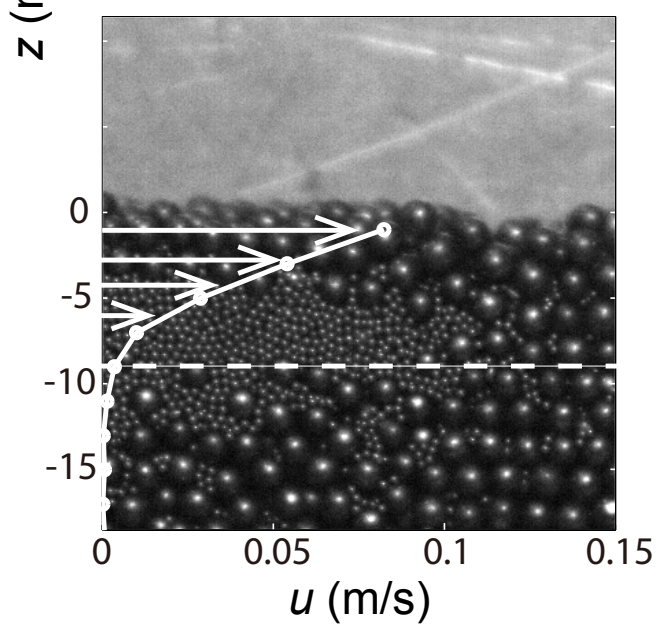

Figure 4: Dynamical process driving the formation of a single stratum for modulated feed rate with $q_{f}=23.6 \mathrm{~cm} / \mathrm{s}$, $t_{f}=5 \mathrm{~s}, q_{s}=2.0 \mathrm{~cm}^{2} / \mathrm{s}$, and $t_{s}=20 \mathrm{~s}$ using $2 \mathrm{~mm}$ (blue) and $0.5 \mathrm{~mm}$ (red) particles. The elapsed time is set to $t=0$ when the fast phase begins, and the origin of the $z$ coordinate is aligned with the free surface. (left column) Images of the entire heap with insets showing the region where the velocity profile is measured. (right column) Images from the high speed camera with an overlay of the instantaneous streamwise velocity profile along the depth coordinate calculated by PTV and averaged over $0.05 \mathrm{~s}$ and across the width of the image. Dashed lines mark the bottom of the flowing layer below which the streamwise velocity is less than $10 \%$ of the surface streamwise velocity. (a) and (b) $t \approx 3 \mathrm{~s}$ and $x / L=0.34$ when the small and large particle layers are about to separate showing the large particle front. (c) and $(\mathrm{d}) t \approx 12 \mathrm{~s}$ and $x / L=0.55$ when the small particle layer is traveling downstream showing the leading edge of the small particle layer. 

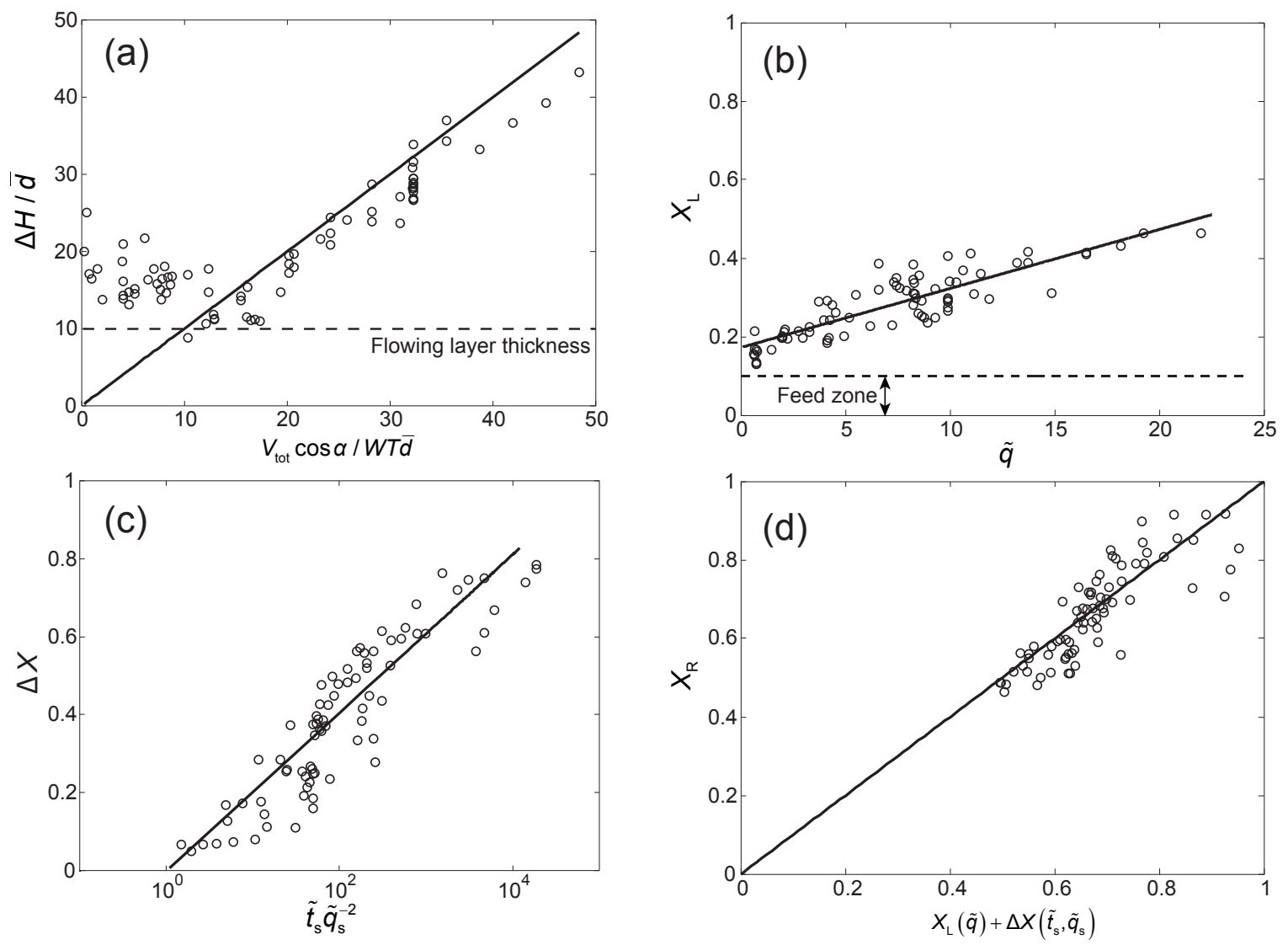

Figure 5:Dependence of stratification on feed rate modulation parameters. (a) Strata thickness $\Delta H$ vs. the predicted volume per feed cycle $V_{\text {tot }}$. The prediction of Eq. (1) is plotted as a line. (b) Left bound of strata $X_{L}$ vs. the nondimensional average feed rate $\tilde{q}$. The line is a linear fit to the data, $X_{L}=0.015 \tilde{q}+0.172$. (c) Interpenetration length $\Delta X$ vs. a non-dimensional parameter related to the slow phase time and feed rate, $\tilde{t}_{s} \tilde{q}_{s}^{-2}$. The line is a fit to the data, $\Delta X=0.088 \ln \left(\tilde{t}_{s} \tilde{q}_{s}^{-2}\right)$. (d) Right bound of strata $X_{R}$ vs. the predicted right bound (solid line) using relations for $X_{L}$ in (b) and $\Delta X$ in (c), $X_{R}=X_{L}(\tilde{q})+\Delta X\left(\tilde{t}_{s}, \tilde{q}_{s}\right)$. 


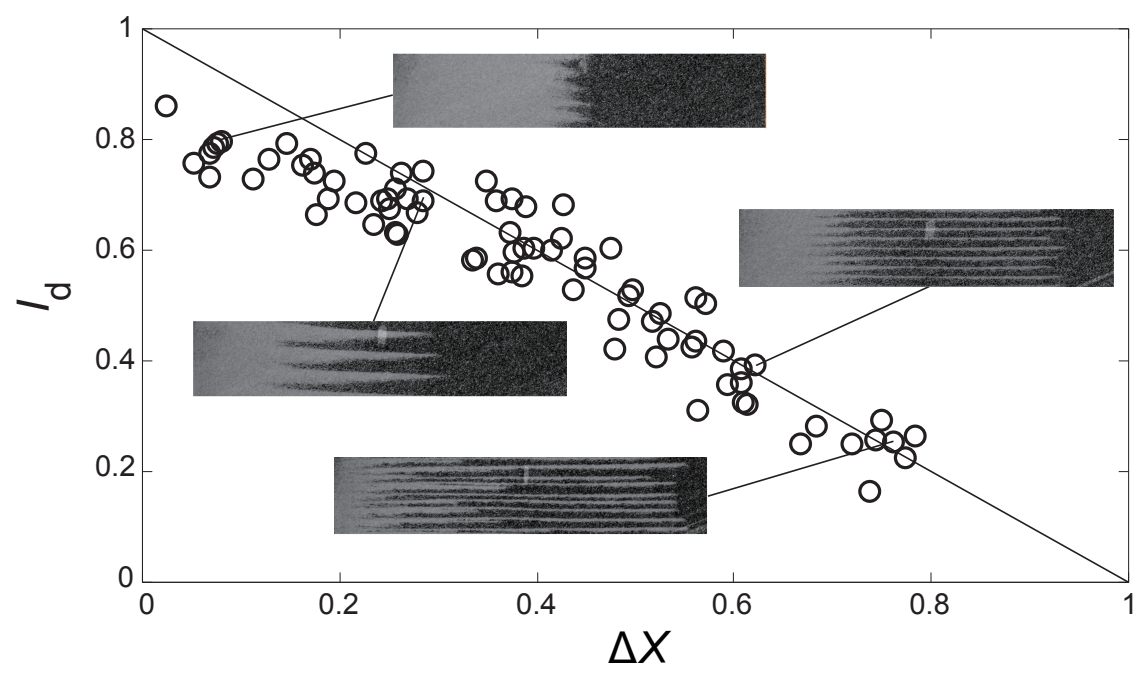

Figure 6: Correlation between the segregation index $I_{d}$ and the interpenetration length $\Delta X$ for the 79 cases in the parametric study compared to $I_{d}=1-\Delta X$ (line). Examples of stratified patterns are shown, where large (small) particles are dark (light) gray (enhanced contrast). 

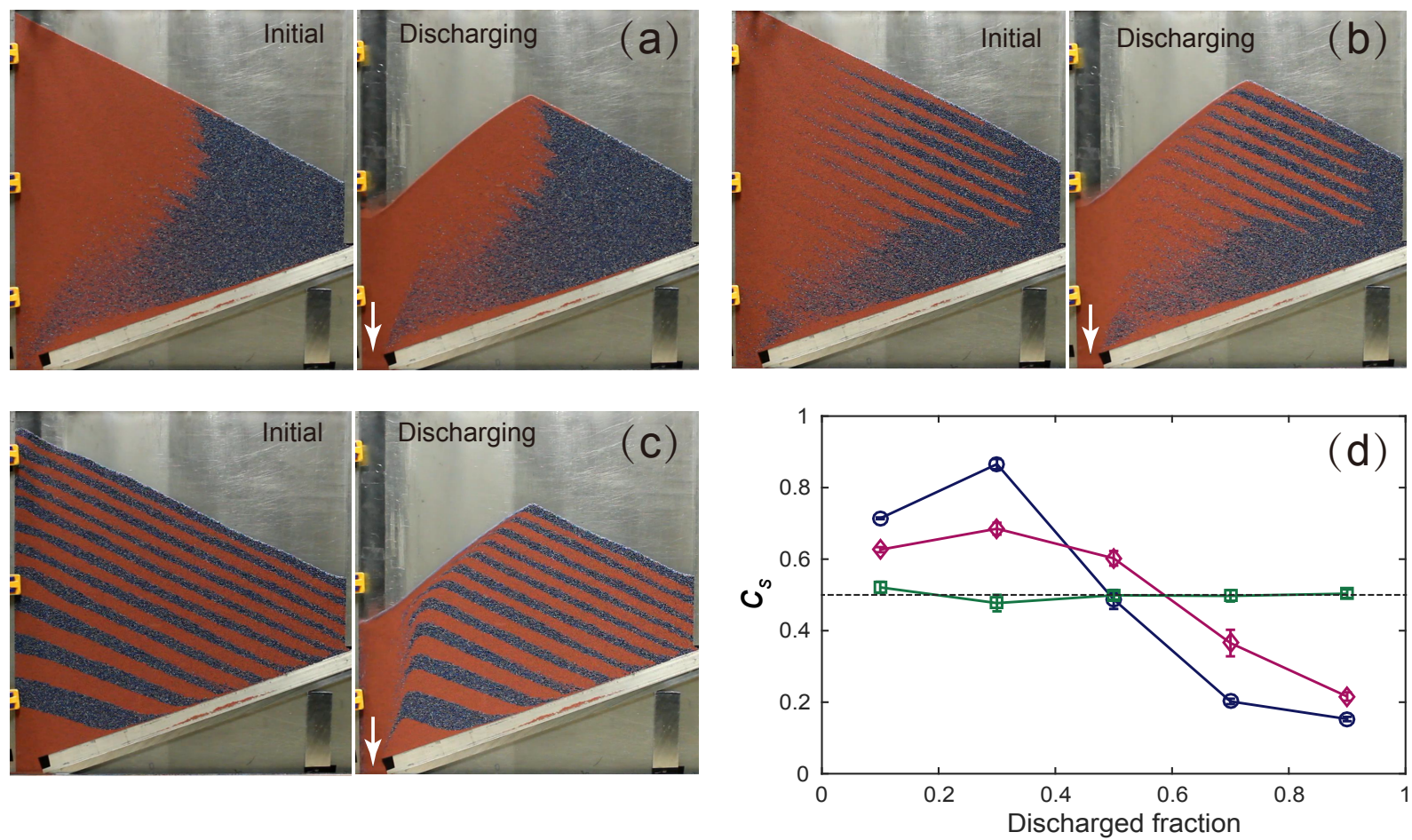

Figure 7: Influence of initial segregation pattern (left images, (a-c)) on discharge (right images, (a-c)) uniformity from a quasi-2D hopper $\left(50 \mathrm{~cm}\right.$ wide, $1.0 \mathrm{~cm}$ thick, bottom inclined at $19^{\circ}$ and with a $3.5 \mathrm{~cm}$ wide exit on the bottom left ). Discharge of (a) initially streamwise segregated pattern generated by filling the hopper with a uniform mixture $\left(0.5 \mathrm{~mm}\right.$ red and $2 \mathrm{~mm}$ diameter blue particles) at a constant feed rate, $q=6.0 \mathrm{~cm}^{2} / \mathrm{s}$; (b) initially stratified pattern generated by filling the hopper using a modulated feed rate with $q_{f}=40.0 \mathrm{~cm}^{2} / \mathrm{s}, t_{f}=2.5 \mathrm{~s}, q_{s}=2.0 \mathrm{~cm}^{2} / \mathrm{s}$, and $t_{s}=23 \mathrm{~s}$; and (c) initially completely stratified pattern generated by feeding large and small particles alternately. (d) Concentration of small particles, $c_{s}$, in the discharge collected for the three cases: streamwise segregated (blue circles), modulated-flow-induced stratified (purple diamonds), and completely stratified (green squares). Error bars indicating the standard deviation are shown (typically smaller than the data symbols). The dashed line indicates $c_{s}=0.5$. 

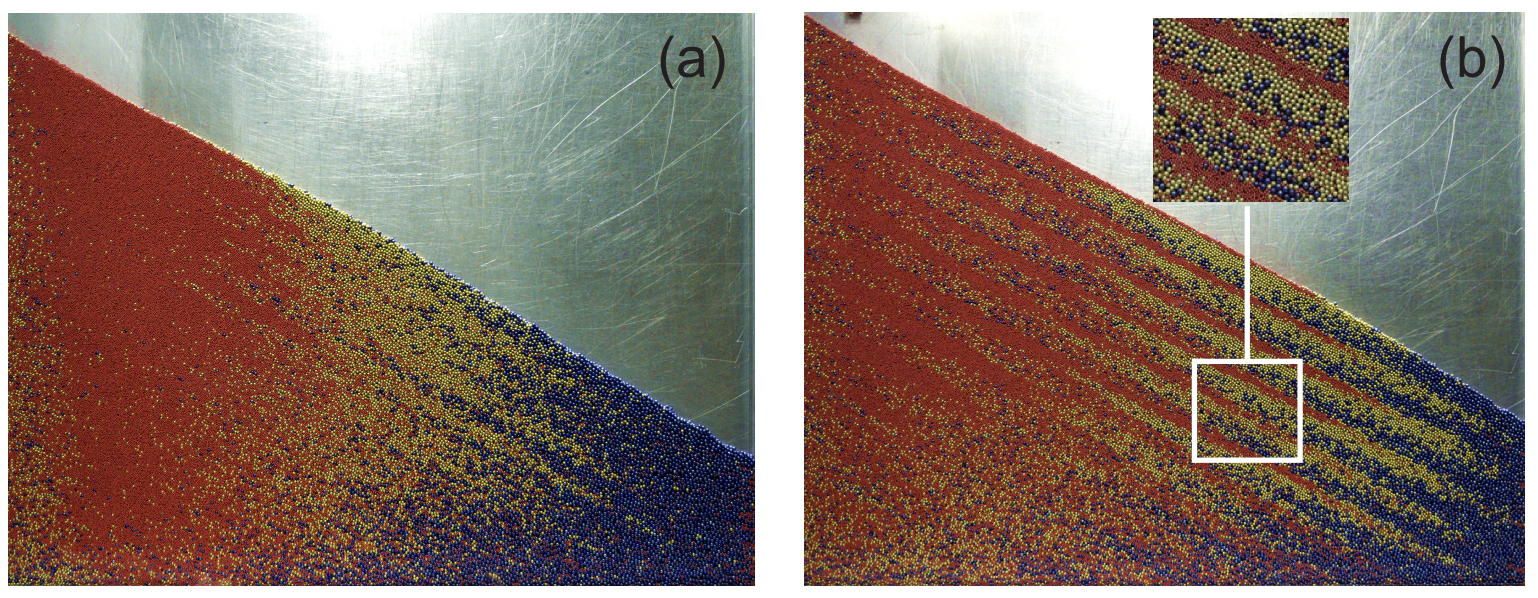

Figure 8: Tridisperese segregation patterns with $1 \mathrm{~mm}$ (red), $2 \mathrm{~mm}$ (gold), and $3 \mathrm{~mm}$ (blue) particles. (a) Steady flow at $q=3.2 \mathrm{~cm}^{2} / \mathrm{s}$. (b) Modulated flow at $\bar{q}=3.2 \mathrm{~cm}^{2} / \mathrm{s}$ for $q_{f}=37.2 \mathrm{~cm}^{2} / \mathrm{s}, t_{f}=4 \mathrm{~s}, q_{s}=1.0 \mathrm{~cm}^{2} / \mathrm{s}$, and $t_{s}=61 \mathrm{~s}$. 
Video
Click here to download Supplementary Material: heap.avi Click here to download Supplementary Material: heap.avi

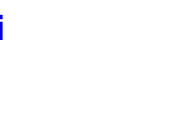

(1) (1) $\sqrt{2}$ (1) $\sqrt{2}$

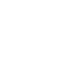

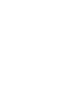

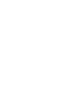
(1) (1)

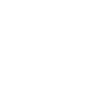
. . (n) (n) (n) (n) (n) (n) (n) (n)

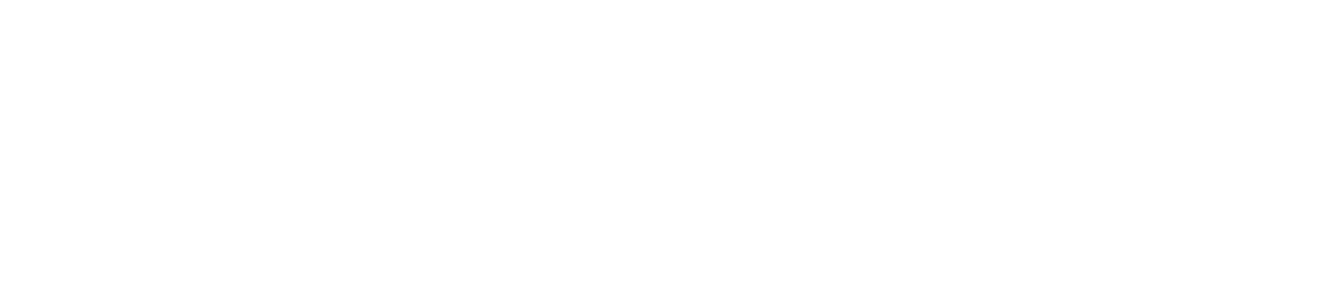
.

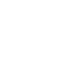

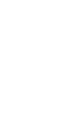

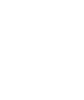

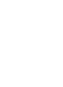
.

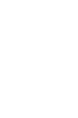

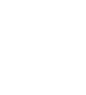

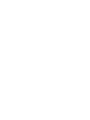

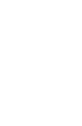

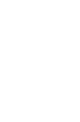
.

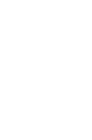

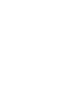

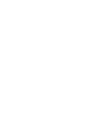

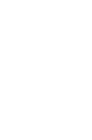

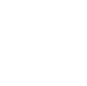

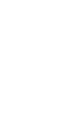



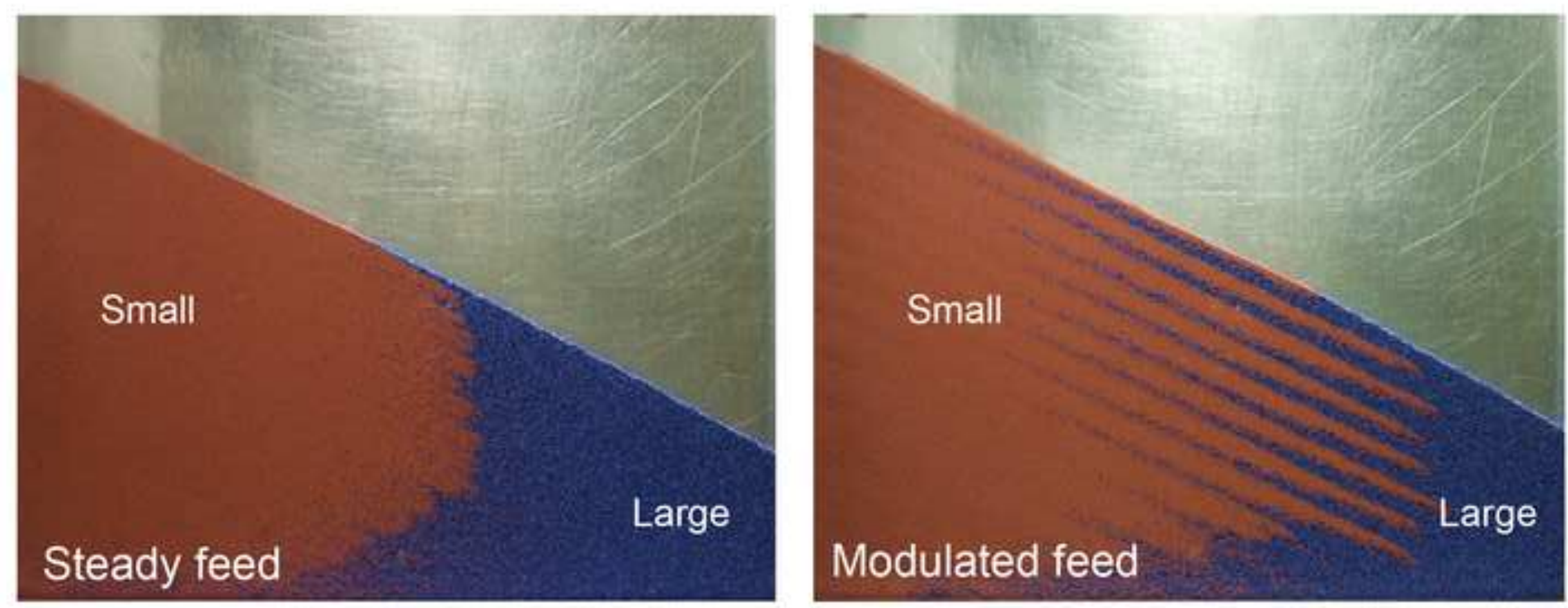